\title{
Web Scale Computing: The Power of Infrastructure as a Service
}

\author{
Peter Vosshall \\ Amazon VP and Distinguished Engineer \\ vosshall@amazon.com
}

\begin{abstract}
Building the right infrastructure that can scale up or down at a moment's notice can be a complicated and expensive task, but it's essential in today's competitive landscape. This applies to an enterprise trying to cut costs, a young business unexpectedly saturated with customer demand, or a research lab wanting to test at scale. There are many challenges when building a reliable, flexible architecture that can manage unpredictable behaviors of today's Internet business. This presentation will outline some of the lessons learned from building one of the world's largest distributed systems, Amazon.com, and the evolution that gave rise to Amazon reselling its infrastructure in the form of Amazon Web Services, allowing anyone to leverage the same robust, scalable, and reliable technology that powers Amazon's business.
\end{abstract}

